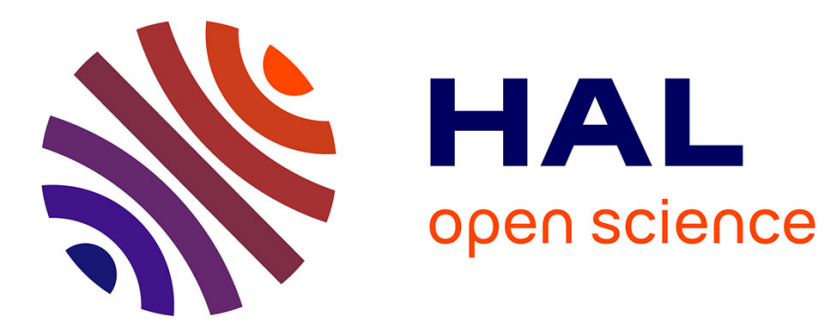

\title{
Hybrid Lower Bound On The MSE Based On The Barankin And Weiss-Weinstein Bounds
}

Chengfang Ren, Jérôme Galy, Eric Chaumette, Pascal Larzabal, Alexandre Renaux

\section{- To cite this version:}

Chengfang Ren, Jérôme Galy, Eric Chaumette, Pascal Larzabal, Alexandre Renaux. Hybrid Lower Bound On The MSE Based On The Barankin And Weiss-Weinstein Bounds. ICASSP: International Conference on Acoustics, Speech, and Signal Processing, May 2013, Vancouver, BC, Canada. pp.55345538, 10.1109/ICASSP.2013.6638722 . hal-00800214

\section{HAL Id: hal-00800214 \\ https://hal-centralesupelec.archives-ouvertes.fr/hal-00800214}

Submitted on 13 Mar 2013

HAL is a multi-disciplinary open access archive for the deposit and dissemination of scientific research documents, whether they are published or not. The documents may come from teaching and research institutions in France or abroad, or from public or private research centers.
L'archive ouverte pluridisciplinaire HAL, est destinée au dépôt et à la diffusion de documents scientifiques de niveau recherche, publiés ou non, émanant des établissements d'enseignement et de recherche français ou étrangers, des laboratoires publics ou privés. 


\title{
HYBRID LOWER BOUND ON THE MSE BASED ON THE BARANKIN AND WEISS-WEINSTEIN BOUNDS
}

\author{
Chengfang Ren ${ }^{(1)}$, Jerome Galy ${ }^{(2)}$, Eric Chaumette ${ }^{(3,4)}$ Pascal Larzabal $^{(4)}$ and Alexandre Renaux $^{(1)}$
}

(1) Universite Paris-Sud/LSS

3, Rue Joliot-Curie, 91192 Gif-sur-Yvette, France. Emails: cren@1ss.supelec.fr,renaux@1ss.supelec.fr

(2) Universite de Montpellier 2/LIRMM

161 rue Ada 34392 Montpellier Cedex 5, France. Email: galy@lirmm.fr

(3) ONERA/The French Aerospace Lab

Chemin de la Huniere Palaiseau Cedex 91123, France. Email: eric.chaumette@onera.fr

(4) Ecole Normale de Cachan/SATIE

61 av. du President Wilson, 94235 Cachan cedex, France. Email: pascal.larzabal@ satie.ens-cachan.fr

\begin{abstract}
This article investigates hybrid lower bounds in order to predict the estimators mean square error threshold effect. A tractable and computationally efficient form is derived. This form combines the Barankin and the Weiss-Weinstein bounds. This bound is applied to a frequency estimation problem for which a closed-form expression is provided. A comparison with results on the hybrid Barankin bound shows the superiority of this new bound to predict the mean square error threshold.
\end{abstract}

Index Terms - Parameter estimation, hybrid bounds, SNR threshold

\section{INTRODUCTION}

In estimation theory, the quality of an estimator is generally measured by its Mean-Squared Error (MSE). In order to quantify its ultimate performance, the MSE is compared to the so-called lower bounds which are independent of estimation technique. Among these bounds, one of the most famous is the Cramér-Rao Bound (CRB) [1][2] due to its easy computation. However, when the observation model is not linear with respect to the parameters to estimate, it is well known that a Signal-to-Noise Ratio (SNR) threshold occurs ([3] p. 273) i.e. large estimation errors appear. Consequently, the knowledge of this particular value for which this threshold appears is fundamental. Unfortunately, the CRB cannot predict this SNR threshold. This is why lower bounds tighter than the CRB have been proposed in the literature. Among these bounds, we distinguish the so-called deterministic lower bounds where the parameters to estimate are assumed to be deterministic unknown e.g. [4][5][6][7][8] and Bayesian lower bounds where the parameters to estimate are assumed to be random with a known a priori Probability Density Function (PDF) e.g. [9][10][11][12][13]. All these bounds have already been successfully applied to various signal processing applications [14].

This work has been partly funded by the European Network of excellence NEWCOM\#
A third family of lower bounds on the MSE called hybrid lower bounds are used to bound the MSE of any estimator which simultaneously estimates both deterministic parameters and random parameters from observations. Indeed, such parametric estimation problems often appear in the literature, for example, the Gaussian generalized linear model [15]. Historically, the first hybrid bound called Hybrid Cramér-Rao Bound (HCRB) has been introduced in 1987 by Rockah and Schultheiss in the context of array shape calibration [16]. Another proof of the HCRB can also be found in [17]. Then, [18] have proposed in 1997 the so-called Hybrid Barankin Bound (HBB) or Reuven-Messer bound and have applied this bound for time-delay estimation in radar signal. Note that, as the classical CRB and Bayesian CRB, the HCRB cannot predict the threshold effect while the HBB does. Recently, [19] have proved three theorems giving necessary and sufficient conditions on the asymptotic achievability of the HCRB. A slight extension of the HCRB where the a priori PDF of the random parameters depends on deterministic parameters has been proposed in [20] and applied to phase estimation in binary phase-shift keying transmission in a non-dataaided context. Finally, a new class of hybrid bound via compression of the sampled centered likelihood-ratio function has been proposed and applied in a frequency estimation context in [21]. Among these aforementioned theoretical results, hybrid lower bounds have been shown to be useful in many applications e.g. refractivity estimation using clutter from sea surface [22], parameters estimation in long-code DS/CDMA systems [23], bearing estimation for deformed towed arrays in the fluid mechanics context [24].

In this paper, we propose a new hybrid lower bound which is based on the Chapman-Robbins bound (a practical approximation of the Barankin bound) for the deterministic parameter and on the Weiss-Weinstein bound for the random parameter. Our motivation comes from the fact that, among the Bayesian bounds, the WeissWeinstein bound is known to be one of the tightest [25]. So, one can expect that the combination of these bounds will lead to a bound tighter than the HBB. The proof is based on the covariance matrix inequality [26]. Particularly, we detail the underlying assumptions on the class of estimators for which the proposed bound can be applied. Last, we give a closed-form expression in a signal processing application with simulation results where a comparison with the Maxi- 
mum A Posteriori / Maximum Likelihood Estimator (MAPMLE) is conducted.

\section{RELATION TO PRIOR WORK}

In the Bayesian context (random parameters only), the WeissWeinstein Bound (WWB) is known to be tighter than the Bayesian Barankin bound [12]. In the hybrid context (where the parameter vector contains both deterministic and random parameters), the HBB [18] has been known so far to be one of the tightest bounds. The purpose of the present paper, by including the WWB, is precisely to offer a new tighter hybrid bound to the signal processing community.

\section{HYBRID LOWER BOUNDS}

\subsection{Background}

Let us first remind the standard assumptions used in the context of hybrid bounds [18]. Consider $\Omega$ an observation space of points $\mathbf{X}$ and let $\boldsymbol{\theta}=\left[\begin{array}{ll}\boldsymbol{\theta}_{d}^{T} & \boldsymbol{\theta}_{r}^{T}\end{array}\right]^{T}$ denotes the hybrid parameter vector to estimate where $\boldsymbol{\theta}_{d} \in \Pi_{d} \subseteq \mathbb{R}^{D}$ is a vector of unknown deterministic parameters and where $\boldsymbol{\theta}_{r} \in \Pi_{r} \subseteq \mathbb{R}^{R}$ is a vector of unknown random parameters. The random parameters are characterized by a prior PDF which is assumed to be independent of $\boldsymbol{\theta}_{d}$. In other words $f\left(\boldsymbol{\theta}_{r} ; \boldsymbol{\theta}_{d}\right)=f\left(\boldsymbol{\theta}_{r}\right)$. Let $f(\mathbf{X}, \boldsymbol{\theta})=f\left(\mathbf{X}, \boldsymbol{\theta}_{r} ; \boldsymbol{\theta}_{d}\right)$ denote the joint PDF of $\mathbf{X}$ and $\boldsymbol{\theta}_{r}$ parameterized by $\boldsymbol{\theta}_{d}$. If $\widehat{\boldsymbol{\theta}}$ is an estimator of $\boldsymbol{\theta}$, then under some mild regularity assumptions, the following covariance inequality holds (e.g. [17],[18] and [26] p. 124) for any real-valued vector $\mathbf{v}(\mathbf{X}, \boldsymbol{\theta})$ with finite second order moment:

$$
\mathbb{E}_{\mathbf{X}, \boldsymbol{\theta}}\left[(\widehat{\boldsymbol{\theta}}-\boldsymbol{\theta})(\widehat{\boldsymbol{\theta}}-\boldsymbol{\theta})^{T}\right] \succeq \mathbf{C V}^{-1} \mathbf{C}^{T},
$$

where $\mathbf{A} \succeq \mathbf{B}$ means that $\mathbf{A}-\mathbf{B}$ is positive semidefinite matrix and where

$$
\mathbf{V}=\mathbb{E}_{\mathbf{X}, \boldsymbol{\theta}}\left[\mathbf{v}(\mathbf{X}, \boldsymbol{\theta}) \mathbf{v}^{T}(\mathbf{X}, \boldsymbol{\theta})\right],
$$

and

$$
\mathbf{C}=\mathbb{E}_{\mathbf{X}, \boldsymbol{\theta}}\left[(\widehat{\boldsymbol{\theta}}-\boldsymbol{\theta}) \mathbf{v}^{T}(\mathbf{X}, \boldsymbol{\theta})\right] .
$$

Note that Eqn. (1) is not a lower bound independent of $\widehat{\boldsymbol{\theta}}$ in general case, since $\mathbf{C}$ depends on $\widehat{\boldsymbol{\theta}}$. However some judicious choices of $\mathbf{v}(\mathbf{X}, \boldsymbol{\theta})$ lead to classical lower bounds on the MSE. For example, if one chooses $\mathbf{v}(\mathbf{X}, \boldsymbol{\theta})=\frac{\partial \ln (f(\mathbf{X}, \boldsymbol{\theta}))}{\partial \boldsymbol{\theta}}$, one obtains the HCRB [16]. On the other hand, if one chooses $\{\mathbf{v}(\mathbf{X}, \boldsymbol{\theta})\}_{i}=$ $\left\{\begin{array}{c}\frac{f\left(\mathbf{X}, \boldsymbol{\theta}+\mathbf{h}_{i}\right)}{f(\mathbf{X}, \boldsymbol{\theta})}-1 \text { if } \boldsymbol{\theta} \in \boldsymbol{\Theta} \\ 0 \text { else }\end{array}\right.$ where $\boldsymbol{\Theta}=\{\boldsymbol{\theta}: f(\mathbf{X}, \boldsymbol{\theta})>0, \mathbf{X} \in \Omega\}$ an
and where $\mathbf{h}_{i}$ are the so called test-points, $i=1, \cdots, N$, one obtains the HBB [18].

\subsection{New hybrid lower bound based on the Barankin and Weiss- Weinstein bounds}

\subsubsection{Notations and assumptions}

For sake of simplicity, we restrict our analysis to the case of a single deterministic parameter and a single random parameter. Consequently, $\boldsymbol{\theta}=\left(\theta_{d} \theta_{r}\right)^{T}$. The multivariate case is cumbersome but straightforward [27]. Regarding the test-points, let us define $\mathbf{h}_{1}=\left(h_{1 d} h_{1 r}\right)^{T}$ and $\mathbf{h}_{2}=\left(0 h_{2 r}\right)^{T}$. Note the fact that the first entry of $\mathbf{h}_{2}$ is equal to 0 is a necessary condition to derive the bound what will be justified in the sequel.
In addition, we have the following assumptions:

1) $\forall \theta_{r} \in \Pi_{r}, f\left(\mathbf{X}, \theta_{r} ; \theta_{d}\right)=0 \Rightarrow f\left(\mathbf{X}, \theta_{r}+h_{r} ; \theta_{d}+h_{d}\right)=0$.

$$
\begin{aligned}
\text { 2) } \forall \theta_{r} \in \Pi_{r}, & \mathbb{E}_{\mathbf{X} \mid \theta ; \theta_{d}}\left[\widehat{\theta}_{d}\right]=\theta_{d}, \\
& \mathbb{E}_{\mathbf{X} \mid \theta_{r} ; \theta_{d}+h_{d}}\left[\widehat{\theta}_{d}\right]=\theta_{d}+h_{d} . \\
\text { 3) } \forall \theta_{d} \in \Pi_{d}, & \mathbb{E}_{\mathbf{X}, \theta_{r} ; \theta_{d}}\left[\widehat{\theta}_{r}-\theta_{r}\right]=0, \\
& \mathbb{E}_{\mathbf{X}, \theta_{r}+h_{r} ; \theta_{d}}\left[\widehat{\theta}_{r}-\left(\theta_{r}+h_{r}\right)\right]=0 .
\end{aligned}
$$

Remark: assumption 1 means that the random parameter support can not be a compact interval; for example the proposed bound does not apply for an uniform prior (as the HBB).

\subsubsection{The proposed bound}

Let us set $\mathbf{v}(\mathbf{X}, \boldsymbol{\theta})=\left(v_{d}(\mathbf{X}, \boldsymbol{\theta}) v_{r}(\mathbf{X}, \boldsymbol{\theta})\right)^{T}$. We propose to use

$$
v_{d}(\mathbf{X}, \boldsymbol{\theta})=\left\{\begin{array}{l}
\frac{f\left(\mathbf{X}, \boldsymbol{\theta}+\mathbf{h}_{1}\right)}{f(\mathbf{X}, \boldsymbol{\theta})}-1 \text { if } \boldsymbol{\theta} \in \mathbf{\Theta}, \\
0 \text { else }
\end{array}\right.
$$

and

$$
v_{r}(\mathbf{X}, \boldsymbol{\theta})=\left\{\begin{array}{l}
\frac{f^{m}\left(\mathbf{X}, \boldsymbol{\theta}+\mathbf{h}_{2}\right)}{f^{m}(\mathbf{X}, \boldsymbol{\theta})}-\frac{f^{1-m}\left(\mathbf{X}, \boldsymbol{\theta}-\mathbf{h}_{2}\right)}{f^{1-m}(\mathbf{X}, \boldsymbol{\theta})} \text { if } \boldsymbol{\theta} \in \mathbf{\Theta} \\
0 \text { else }
\end{array},\right.
$$

where $0<m<1$. Note that:

- $v_{r}(\mathbf{X}, \boldsymbol{\theta}) \underset{m \rightarrow 1}{\rightarrow} \frac{f\left(\mathbf{X}, \boldsymbol{\theta}+\mathbf{h}_{2}\right)}{f(\mathbf{X}, \boldsymbol{\theta})}-1$, which is the choice of $\mathbf{v}(\mathbf{X}, \boldsymbol{\theta})$ leading to a particular HBB with two test-points.

- If the problem is reduced to random parameter only i.e. $\boldsymbol{\theta}=\theta_{r}$ and $\mathbf{v}(\mathbf{X}, \boldsymbol{\theta})=v_{r}(\mathbf{X}, \boldsymbol{\theta})$, then Eqn. (1) reduces to the Bayesian Weiss-Weinstein Bound ([12] Eqn. (19)).

In this case, plugging Eqn. (4) and (5) in Eqn. (2) and (3) will lead to the proposed hybrid lower bound on the MSE. Straightforwardly the elements of matrix $\mathbf{V}$ are given by

$$
\{\mathbf{V}\}_{1,1}=\mu\left(2, \mathbf{h}_{1}\right)-1
$$

$$
\{\mathbf{V}\}_{2,2}=\mu\left(2 m, \mathbf{h}_{2}\right)+\mu\left(2-2 m,-\mathbf{h}_{2}\right)-2 \mu\left(m, 2 \mathbf{h}_{2}\right),
$$
and

$$
\begin{aligned}
\{\mathbf{V}\}_{1,2}= & \{\mathbf{V}\}_{2,1}=\eta\left(m, \mathbf{h}_{1}, \mathbf{h}_{2}\right)-\eta\left(1-m, \mathbf{h}_{1},-\mathbf{h}_{2}\right) \\
& -\mu\left(m, \mathbf{h}_{2}\right)+\mu\left(1-m,-\mathbf{h}_{2}\right)
\end{aligned}
$$

where

$$
\mu(m, \mathbf{h})=\mathbb{E}_{\mathbf{X}, \boldsymbol{\theta}}\left[\frac{f^{m}(\mathbf{X}, \boldsymbol{\theta}+\mathbf{h})}{f^{m}(\mathbf{X}, \boldsymbol{\theta})}\right],
$$

$$
\eta\left(\alpha, \mathbf{h}_{1}, \mathbf{h}_{2}\right)=\mathbb{E}_{\mathbf{X}, \boldsymbol{\theta}}\left[\frac{f\left(\mathbf{X}, \boldsymbol{\theta}+\mathbf{h}_{1}\right)}{f(\mathbf{X}, \boldsymbol{\theta})} \frac{f^{\alpha}\left(\mathbf{X}, \boldsymbol{\theta}+\mathbf{h}_{2}\right)}{f^{\alpha}(\mathbf{X}, \boldsymbol{\theta})}\right] .
$$

Concerning the elements of matrix $\mathbf{C}$, one has to prove that they are independent of $\widehat{\boldsymbol{\theta}}$ in order to obtain a lower bound independent of the estimation scheme. After calculations detailed on Appendix, one obtains

$$
\{\mathbf{C}\}_{1,1}=h_{1 d},\{\mathbf{C}\}_{2,1}=h_{1 r},\{\mathbf{C}\}_{1,2}=0
$$

and

$$
\{\mathbf{C}\}_{2,2}=h_{2 r} \mu\left(m, \mathbf{h}_{2}\right) .
$$

Finally, the lower bound is given by

$$
\max _{\mathbf{h}_{1} \in \Pi_{d} \times \Pi_{r}, \mathbf{h}_{2} \in\{0\} \times \Pi_{r}, 0<m<1}\left\{\mathbf{C V}^{-1} \mathbf{C}^{T}\right\} .
$$




\section{APPLICATION AND SIMULATION}

\subsection{Example of application}

We consider the frequency estimation problem given in [21], where the observation model is:

$$
\mathbf{x}=s e^{j \varphi} \mathbf{b}(\omega)+\mathbf{n},
$$

and $\mathbf{x} \in \mathbb{C}^{P}$ is the observation vector, $\mathbf{b}(\omega)=\left[1 e^{j \omega} \cdots e^{j(P-1) \omega}\right]^{T}$ is a normalized cisoid signal and the noise $\mathbf{n}$ is assumed to be complex Gaussian circular centred with covariance matrix $\sigma_{n}^{2} \mathbf{I}$.

We assume that the phase $\varphi \in]-\pi ; \pi]$ is known, the amplitude $s \in \mathbb{R}^{+}$is a deterministic unknown parameter and the frequency $\omega$ is a random unknown parameter with a priori Gaussian centred with variance $\sigma_{\omega}^{2}$. Therefore the unknown vector of parameters to estimate is $\boldsymbol{\theta}=[s \omega]^{T}$.

The conditional density of probability of $\mathbf{x} \mid \omega ; s$ is

$$
f_{\mathbf{x} \mid \omega ; s}(\mathbf{x} \mid \omega ; s)=\frac{e^{-\frac{1}{\sigma_{n}^{2}}\left\|\mathbf{x}-s e^{j \varphi} \mathbf{b}(\omega)\right\|^{2}}}{\left(\pi \sigma_{n}^{2}\right)^{P}} .
$$

We assume that $\omega$ is independent of $s$, so the joint law is given by:

$$
\begin{aligned}
f_{\mathbf{x}, \omega ; s}(\mathbf{x}, \omega ; s) & =f_{\mathbf{x} \mid \omega ; s}(\mathbf{x} \mid \omega ; s) f_{\omega}(\omega) \\
& =\frac{e^{-\frac{1}{\sigma_{n}^{2}}\left\|\mathbf{x}-s e^{j \varphi} \mathbf{b}(\omega)\right\|^{2}-\frac{\omega^{2}}{2 \sigma_{\omega}^{2}}}}{\left(\pi \sigma_{n}^{2}\right)^{P} \sqrt{2 \pi \sigma_{\omega}^{2}}} .
\end{aligned}
$$

To compute the new hybrid bound, we need to calculate the expression (9) and (10). Some cumbersome but not difficult calculus [27] yields the following expressions for any $\mathbf{h}=\left[h_{s} h_{\omega}\right]^{T}$ and $m$ :

$$
\mu(m, \mathbf{h})=e^{\frac{m(m-1)}{\sigma_{n}^{2}}\left(\sum_{t=0}^{P-1}\left|\left(s+h_{s}\right) e^{j h \omega t}-s\right|^{2}\right)+\frac{m(m-1) h_{\omega}^{2}}{2 \sigma_{\omega}^{2}}}
$$

and for any $\mathbf{h}_{1}=\left[h_{1 s} h_{1 \omega}\right]^{T}$ and $\mathbf{h}_{2}=\left[0 h_{2 \omega}\right]^{T}$ and $\alpha$ :

$\eta\left(\alpha, \mathbf{h}_{1}, \mathbf{h}_{2}\right)=\frac{e^{\frac{1}{\sigma_{n}^{2}}\left(\sum_{t=0}^{P-1}\left|\left(s+h_{1 s}\right) e^{j h_{1 \omega} t}+\alpha s e^{j h_{2 \omega} t}-\alpha s\right|^{2}\right)+\alpha \frac{h_{2 \omega} h_{1 \omega}}{\sigma_{\omega}^{2}}}}{e^{\frac{1}{\sigma_{n}^{2}} P\left(s+h_{1 s}\right)^{2}}}$

\subsection{Simulation}

In the application example considered: $s=1, \varphi=\frac{\pi}{4}, \sigma_{\omega}^{2}=$ $\frac{1}{2}$ and $P=2^{5}$. From [15], the HCRB is $2 \times 2$ diagonal matrix with entries $\{\mathbf{H C R B}\}_{1,1}=\frac{\sigma_{n}^{2}}{2 P}$ and $\{\mathbf{H C R B}\}_{2,2}=$ $\left(\frac{2 s^{2}}{\sigma_{n}^{2}}\left(\frac{P(P+1)(2 P+1)}{6}-P^{2}\right)+\frac{1}{\sigma_{\omega}^{2}}\right)^{-1}$. The HBB and the new bound are computed with $\mathbf{h}_{1} \in[-1 ; 1] \times\{0\}$ where the sampling interval for the first component is $\delta h_{s}=0.01$ and $\mathbf{h}_{2} \in\{0\} \times\left[-\frac{3}{2} ; \frac{3}{2}\right]$ where the sampling interval for the second component is $\delta h_{\omega}=\frac{3}{2^{8}}$. Last, the MAPMLE is obtained by searching the best candidate $s \in[0 ; 2]$ and $\omega \in\left[-\frac{3}{2} ; \frac{3}{2}\right]$ maximizing the joint PDF $f_{\mathbf{x}, \omega ; s}(\mathbf{x}, \omega ; s)$.

The empirical MSE of the MAPMLE is assessed with 1000 MonteCarlo trials.

Since in our application case the observation model is linear in $s$ and non linear in $\omega$, we only plot on the figure (1) the HCRB, the HBB, the proposed bound denoted HBWWB, and the empirical MSE of the MAPMLE for the random parameter $\omega$ which is the only one to exhibit a SNR threshold phenomenon.

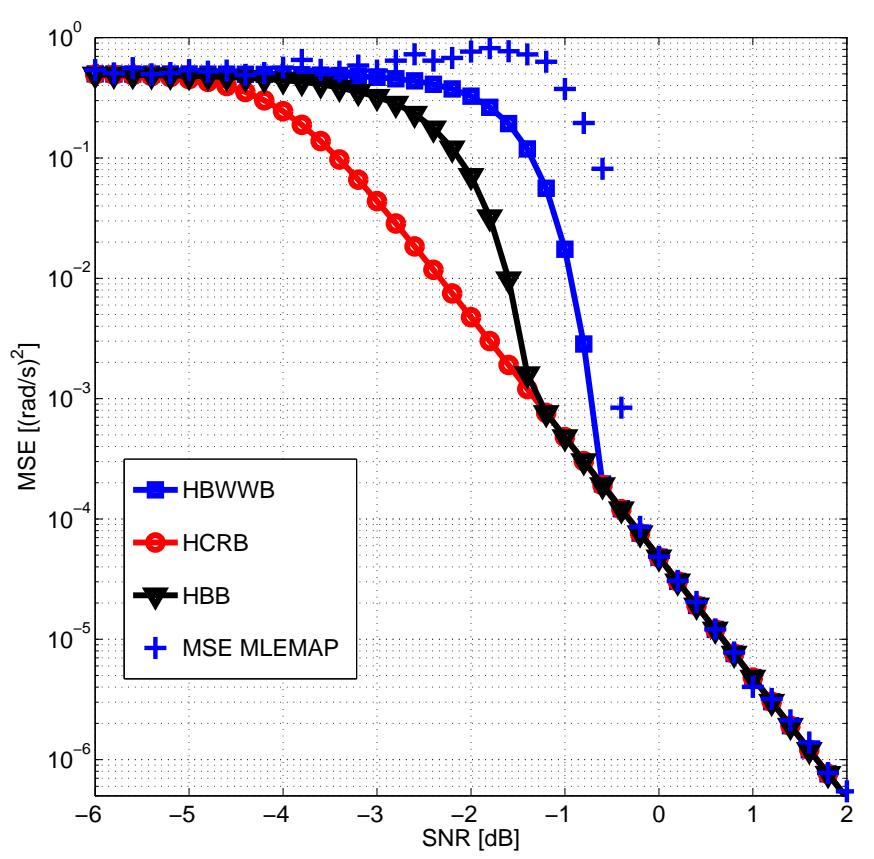

Fig. 1. Comparison of MSE hybrid lower bounds versus SNR

\section{CONCLUSION}

In this paper, a hybrid lower bound on the mean square error based on the Barankin bound and on the Weiss-Weinstein bound has been developed. This bound can be applied to the same class of estimators as the hybrid Barankin bound but exhibits a better SNR threshold prediction.

\section{APPENDIX}

Concerning the element $\{\mathbf{C}\}_{1,1}$, we have

$$
\begin{aligned}
\{\mathbf{C}\}_{1,1} & =\mathbb{E}_{\mathbf{X}, \boldsymbol{\theta}}\left[\left(\widehat{\theta}_{d}-\theta_{d}\right) v_{d}(\mathbf{X}, \boldsymbol{\theta})\right] \\
& =\int_{\Pi_{r}} \int_{\Omega}\left(\widehat{\theta}_{d}-\theta_{d}\right)\left(\frac{f\left(\mathbf{X}, \boldsymbol{\theta}+\mathbf{h}_{1}\right)}{f(\mathbf{X}, \boldsymbol{\theta})}-1\right) f(\mathbf{X}, \boldsymbol{\theta}) d \mathbf{X} d \theta_{r} \\
& =\int_{\Pi_{r}}\left(\begin{array}{c}
f\left(\theta_{r}+h_{1 r}\right) \int_{\Omega}\left(\widehat{\theta}_{d}-\theta_{d}\right) \\
\times f\left(\mathbf{X} \mid \theta_{r}+h_{1 r} ; \theta_{d}+h_{1 d}\right) d \mathbf{X} \\
-f\left(\theta_{r}\right) \int_{\Omega}\left(\widehat{\theta}_{d}-\theta_{d}\right) f\left(\mathbf{X} \mid \theta_{r} ; \theta_{d}\right) d \mathbf{X}
\end{array}\right) d \theta_{r} .
\end{aligned}
$$

By using assumption 2, one obtains

$$
\int_{\Omega}\left(\widehat{\theta}_{d}-\theta_{d}\right) f\left(\mathbf{X} \mid \theta_{r} ; \theta_{d}\right) d \mathbf{X}=0,
$$

and

$$
\begin{gathered}
\int_{\Omega}\left(\widehat{\theta}_{d}-\left(\theta_{d}+h_{1 d}\right)\right) f\left(\mathbf{X} \mid \theta_{r}+h_{1 r} ; \theta_{d}+h_{1 d}\right) d \mathbf{X}=0, \\
\mathbb{1} \\
\int_{\Omega}\left(\widehat{\theta}_{d}-\theta_{d}\right) f\left(\mathbf{X} \mid \theta_{r}+h_{1 r} ; \theta_{d}+h_{1 d}\right) d \mathbf{X}=h_{1 d} .
\end{gathered}
$$


Thus, plugging Eqn. (20) and (21) in Eqn. (19), we have

$$
\{\mathbf{C}\}_{1,1}=h_{1 d} \int_{\Pi_{r}} f\left(\theta_{r}+h_{1 r}\right) d \theta_{r}=h_{1 d} .
$$

Regarding the element $\{\mathbf{C}\}_{2,1}$, we have

$$
\begin{aligned}
\{\mathbf{C}\}_{2,1} & =\mathbb{E}_{\mathbf{X}, \boldsymbol{\theta}}\left[\left(\widehat{\theta}_{r}-\theta_{r}\right) v_{d}(\mathbf{X}, \boldsymbol{\theta})\right] \\
& =\int_{\Pi_{r}} \int_{\Omega}\left(\widehat{\theta}_{r}-\theta_{r}\right)\left(\frac{f\left(\mathbf{X}, \boldsymbol{\theta}+\mathbf{h}_{1}\right)}{f(\mathbf{X}, \boldsymbol{\theta})}-1\right) f(\mathbf{X}, \boldsymbol{\theta}) d \mathbf{X} d \theta_{r} \\
& =\left(\begin{array}{c}
\int_{\Pi_{r}} \int_{\Omega}\left(\widehat{\theta}_{r}-\theta_{r}\right) f\left(\mathbf{X}, \boldsymbol{\theta}+\mathbf{h}_{1}\right) d \mathbf{X} d \theta_{r} \\
\left.-\int_{\Pi_{r}} \int_{\Omega}\left(\widehat{\theta}_{r}-\theta_{r}\right) f(\mathbf{X}, \boldsymbol{\theta}) d \mathbf{X} d \theta_{r}\right)
\end{array}\right.
\end{aligned}
$$

By using the assumption 3, one obtains

$$
\int_{\Pi_{r}} \int_{\Omega}\left(\widehat{\theta}_{r}-\theta_{r}\right) f(\mathbf{X}, \boldsymbol{\theta}) d \mathbf{X} d \theta_{r}=0
$$

and

$$
\begin{aligned}
& \int_{\Pi_{r}} \int_{\Omega}\left(\widehat{\theta}_{r}-\left(\theta_{r}+h_{1 r}\right)\right) f\left(\mathbf{X}, \boldsymbol{\theta}+\mathbf{h}_{1}\right) d \mathbf{X} d \theta_{r}=0 \\
& \int_{\Pi_{r}} \int_{\Omega}\left(\widehat{\theta}_{r}-\theta_{r}\right) f\left(\mathbf{X}, \boldsymbol{\theta}+\mathbf{h}_{1}\right) d \mathbf{X} d \theta_{r}= \\
& h_{1 r} \int_{\Pi_{r}} \int_{\Omega} f\left(\mathbf{X} \mid \theta_{r}+h_{1 r} ; \theta_{d}+h_{1 d}\right) f\left(\theta_{r}+h_{1 r}\right) d \mathbf{X} d \theta_{r} .
\end{aligned}
$$

By substitution $\theta_{r}^{\prime}=\theta_{r}+h_{1 r}$ and by assumption 1, the integer space is still $\Pi_{r}$, and

$$
\begin{aligned}
\{\mathbf{C}\}_{2,1} & =h_{1 r} \int_{\Pi_{r}} \int_{\Omega} f\left(\mathbf{X} \mid \theta_{r}^{\prime} ; \theta_{d}+h_{1 d}\right) f\left(\theta_{r}^{\prime}\right) d \mathbf{X} d \theta_{r}^{\prime} \\
& =h_{1 r} .
\end{aligned}
$$

Before calculating $\{\mathbf{C}\}_{1,2}$ and $\{\mathbf{C}\}_{2,2}$, we give a preliminary result: for any real-valued function $g\left(\mathbf{X}, \theta_{d}\right)$ defined on $\Omega \times \Pi_{d}$ and for any $\mathbf{h}=\left(0 h_{r}\right)^{T}$ where $h_{r} \in \Pi_{r}$, one has

$$
\begin{aligned}
& \int_{\Pi_{r}} g\left(\mathbf{X}, \theta_{d}\right)\left(\frac{f^{m}(\mathbf{X}, \boldsymbol{\theta}+\mathbf{h})}{f^{m}(\mathbf{X}, \boldsymbol{\theta})}-\frac{f^{1-m}(\mathbf{X}, \boldsymbol{\theta}-\mathbf{h})}{f^{1-m}(\mathbf{X}, \boldsymbol{\theta})}\right) f(\mathbf{X}, \boldsymbol{\theta}) d \theta_{r} \\
& =g\left(\mathbf{X}, \theta_{d}\right) \int_{\Pi_{r}}\left(\begin{array}{c}
f^{m}(\mathbf{X}, \boldsymbol{\theta}+\mathbf{h}) f^{1-m}(\mathbf{X}, \boldsymbol{\theta}) \\
-f^{1-m}(\mathbf{X}, \boldsymbol{\theta}-\mathbf{h}) f^{m}(\mathbf{X}, \boldsymbol{\theta})
\end{array}\right) d \theta_{r} .
\end{aligned}
$$

Note that

$$
\left.\begin{array}{c}
\int_{\Pi_{r}}\left(\begin{array}{c}
f^{m}(\mathbf{X}, \boldsymbol{\theta}+\mathbf{h}) f^{1-m}(\mathbf{X}, \boldsymbol{\theta}) \\
-f^{1-m}(\mathbf{X}, \boldsymbol{\theta}-\mathbf{h}) f^{m}(\mathbf{X}, \boldsymbol{\theta})
\end{array}\right) d \theta_{r}= \\
\left(\int_{\Pi_{r}}^{f^{m}\left(\mathbf{X}, \theta_{r}+h_{r} ; \theta_{d}\right) f^{1-m}\left(\mathbf{X}, \theta_{r} ; \theta_{d}\right) d \theta_{r}}\right. \\
-\int_{\Pi_{r}}^{\Pi^{1-m}}\left(\mathbf{X}, \theta_{r}-h_{r} ; \theta_{d}\right) f^{m}\left(\mathbf{X}, \theta_{r} ; \theta_{d}\right) d \theta_{r}
\end{array}\right)
$$

Let us study the first integral. By substituting $\theta_{r}^{\prime}=\theta_{r}+h_{r}$, the integer space is still $\Pi_{r}$ by assumption 1 and then,

$$
\begin{aligned}
\int_{\Pi_{r}} f^{m}\left(\mathbf{X}, \theta_{r}+h_{r} ; \theta_{d}\right) f^{1-m}\left(\mathbf{X}, \theta_{r} ; \theta_{d}\right) d \theta_{r} \\
\quad=\int_{\Pi_{r}} f^{m}\left(\mathbf{X}, \theta_{r}^{\prime} ; \theta_{d}\right) f^{1-m}\left(\mathbf{X}, \theta_{r}^{\prime}-h_{r} ; \theta_{d}\right) d \theta_{r}^{\prime},
\end{aligned}
$$

Thus, using (29) into (27), one obtains

$\int_{\Pi_{r}} g\left(\mathbf{X}, \theta_{d}\right)\left(\frac{f^{m}(\mathbf{X}, \boldsymbol{\theta}+\mathbf{h})}{f^{m}(\mathbf{X}, \boldsymbol{\theta})}-\frac{f^{1-m}(\mathbf{X}, \boldsymbol{\theta}-\mathbf{h})}{f^{1-m}(\mathbf{X}, \boldsymbol{\theta})}\right) f(\mathbf{X}, \boldsymbol{\theta}) d \theta_{r}$ $=0$ a.e. $\mathbf{X} \in \Omega$ and for every $\theta_{d} \in \Pi_{d}$

Remarks:

- This result is an extension of Eqn. (1) in [12] when the joint PDF depends on $\theta_{d}$.

- If we chose $\mathbf{h}=\left(h_{d} h_{r}\right)$ with $h_{d} \neq 0$ in this premilinary result, then Eqn. (30) would depend on $\mathbf{X}$. Consequently, we would find that $\{\mathbf{C}\}_{1,2}$ and $\{\mathbf{C}\}_{2,2}$ would depend on $\widehat{\boldsymbol{\theta}}$.

Now, concerning $\{\mathbf{C}\}_{1,2}$, one has

$$
\begin{aligned}
\{\mathbf{C}\}_{1,2} & =\mathbb{E}_{\mathbf{X}, \boldsymbol{\theta}}\left[\left(\widehat{\theta}_{d}-\theta_{d}\right) v_{r}(\mathbf{X}, \boldsymbol{\theta})\right] \\
& =\int_{\Omega}\left(\widehat{\theta}_{d}-\theta_{d}\right) \int_{\Pi_{r}}\left(\begin{array}{c}
\frac{f^{m}\left(\mathbf{X}, \boldsymbol{\theta}+\mathbf{h}_{2}\right)}{f^{m}(\mathbf{X}, \boldsymbol{\theta})} \\
-\frac{f^{1-m\left(\mathbf{X} \boldsymbol{\theta}-\mathbf{h}_{2}\right)}}{f^{1-m}(\mathbf{X}, \boldsymbol{\theta})}
\end{array}\right) f(\mathbf{X}, \boldsymbol{\theta}) d \theta_{r} d \mathbf{X} \\
& =0,
\end{aligned}
$$

using Eqn. (30) with $g\left(\mathbf{X}, \theta_{d}\right)=\widehat{\theta}_{d}-\theta_{d}$.

Finally, concerning $\{\mathbf{C}\}_{2,2}$, one has

$$
\begin{aligned}
\{\mathbf{C}\}_{2,2} & =\mathbb{E}_{\mathbf{X}, \boldsymbol{\theta}}\left[\left(\widehat{\theta}_{r}-\theta_{r}\right) v_{r}(\mathbf{X}, \boldsymbol{\theta})\right] \\
& =\iint_{\Omega} \int_{\Pi_{r}}\left(\widehat{\theta}_{r}-\theta_{r}\right)\left(\begin{array}{c}
\frac{f^{m}\left(\mathbf{X}, \boldsymbol{\theta}+\mathbf{h}_{2}\right)}{f^{m}(\mathbf{X}, \boldsymbol{\theta})} \\
-\frac{f^{1-m}\left(\mathbf{X}, \boldsymbol{\theta}-\mathbf{h}_{2}\right)}{f^{1-m}(\mathbf{X}, \boldsymbol{\theta})}
\end{array}\right) f(\mathbf{X}, \boldsymbol{\theta}) d \theta_{r} d \mathbf{X} \\
& =\int_{\Omega} \int_{\Pi_{r}} \theta_{r}\left(\begin{array}{c}
\frac{f^{1-m}\left(\mathbf{X}, \boldsymbol{\theta}-\mathbf{h}_{2}\right)}{f^{1-m}(\mathbf{X}, \boldsymbol{\theta})} \\
-\frac{f^{m}\left(\mathbf{X}, \boldsymbol{\theta}+\mathbf{h}_{2}\right)}{f^{m}(\mathbf{X}, \boldsymbol{\theta})}
\end{array}\right) f(\mathbf{X}, \boldsymbol{\theta}) d \theta_{r} d \mathbf{X}
\end{aligned}
$$

by using Eqn. (30) with $g\left(\mathbf{X}, \theta_{d}\right)=\widehat{\theta}_{r}$. Let us study

$$
\begin{aligned}
& \int_{\Pi_{r}} \theta_{r} f^{1-m}\left(\mathbf{X}, \boldsymbol{\theta}-\mathbf{h}_{2}\right) f^{m}(\mathbf{X}, \boldsymbol{\theta}) d \theta_{r}= \\
& \int_{\Pi_{r}} \theta_{r} f^{1-m}\left(\mathbf{X}, \theta_{r}-h_{2 r} ; \theta_{d}\right) f^{1}\left(\mathbf{X}, \theta_{r} ; \theta_{d}\right) d \theta_{r} .
\end{aligned}
$$

By substitution $\theta_{r}^{\prime}=\theta_{r}-h_{2 r}$, the integer space for $\theta_{r}^{\prime}$ is still $\Pi_{r}$ by assumption 1 and we have

$$
\begin{aligned}
& \int_{\Pi_{r}} \theta_{r} f^{1-m}\left(\mathbf{X}, \boldsymbol{\theta}-\mathbf{h}_{2}\right) f^{m}(\mathbf{X}, \boldsymbol{\theta}) d \theta_{r} \\
& =\int_{\Pi_{r}}\left(\theta_{r}^{\prime}+h_{2 r}\right) f^{1-m}\left(\mathbf{X}, \theta_{r}^{\prime} ; \theta_{d}\right) f^{m}\left(\mathbf{X}, \theta_{r}^{\prime}+h_{2 r} ; \theta_{d}\right) d \theta_{r}^{\prime} \\
& =\int_{\Pi_{r}} \theta_{r}^{\prime} f^{m}\left(\mathbf{X}, \theta_{r}^{\prime}+h_{2 r} ; \theta_{d}\right) f^{1-m}\left(\mathbf{X}, \theta_{r}^{\prime} ; \theta_{d}\right) d \theta_{r}^{\prime} \\
& \quad+h_{2 r} \int_{\Pi_{r}} f^{m}\left(\mathbf{X}, \theta_{r}^{\prime}+h_{2 r} ; \theta_{d}\right) f^{1-m}\left(\mathbf{X}, \theta_{r}^{\prime} ; \theta_{d}\right) d \theta_{r}^{\prime} .
\end{aligned}
$$

Thus, plugging Eqn. (33) in (31), one has

$$
\{\mathbf{C}\}_{2,2}=h_{2 r} \mathbb{E}_{X, \boldsymbol{\theta}}\left[\frac{f^{m}\left(\mathbf{X}, \boldsymbol{\theta}+\mathbf{h}_{2}\right)}{f^{m}(\mathbf{X}, \boldsymbol{\theta})}\right] .
$$




\section{REFERENCES}

[1] H. Cramér, Mathematical Methods of Statistics, ser. Princeton Mathematics. New-York: Princeton University Press, Sep. 1946, vol. 9 .

[2] H. L. Van Trees, Detection, Estimation and Modulation Theory. New-York, NY, USA: John Wiley \& Sons, 1968, vol. 1.

[3] — Detection, Estimation and Modulation Theory: RadarSonar Signal Processing and Gaussian Signals in Noise. New-York, NY, USA: John Wiley \& Sons, Sep. 2001, vol. 3.

[4] D. G. Chapman and H. Robbins, "Minimum variance estimation without regularity assumptions," The Annals of Mathematical Statistics, vol. 22, no. 4, pp. 581-586, Dec. 1951.

[5] R. J. McAulay and L. P. Seidman, "A useful form of the Barankin lower bound and its application to PPM threshold analysis," IEEE Transactions on Information Theory, vol. 15, no. 2, pp. 273-279, Mar. 1969.

[6] J. S. Abel, "A bound on mean square estimate error," IEEE Transactions on Information Theory, vol. 39, no. 5, pp. 16751680, Sep. 1993.

[7] K. Todros and J. Tabrikian, "General classes of performance lower bounds for parameter estimation - part I: non-Bayesian bounds for unbiased estimators," IEEE Transactions on Information Theory, vol. 56, no. 10, pp. 5045-5063, Oct. 2010.

[8] E. Chaumette, J. Galy, A. Quinlan, and P. Larzabal, "A new Barankin bound approximation for the prediction of the threshold region performance of maximum likelihood estimators," IEEE Transactions on Signal Processing, vol. 56, no. 11, pp. 5319-5333, Nov. 2008.

[9] J. Ziv and M. Zakai, "Some lower bounds on signal parameter estimation," IEEE Transactions on Information Theory, vol. 15, no. 3, pp. 386-391, May 1969.

[10] K. L. Bell, Y. Steinberg, Y. Ephraim, and H. L. Van Trees, "Extended Ziv-Zakaï lower bound for vector parameter estimation," IEEE Transactions on Information Theory, vol. 43, no. 2, pp. 624-637, Mar. 1997.

[11] A. Renaux, P. Forster, P. Larzabal, and C. D. Richmond, "The Bayesian Abel bound on the mean square error," in Proc. of IEEE International Conference on Acoustics, Speech, and Signal Processing (ICASSP), vol. 3, Toulouse, FR, May 2006, pp. 9-12.

[12] E. Weinstein and A. J. Weiss, "A general class of lower bounds in parameter estimation," IEEE Transactions on Information Theory, vol. 34, no. 2, pp. 338-342, Mar. 1988.

[13] K. Todros and J. Tabrikian, "General classes of performance lower bounds for parameter estimation - part II: Bayesian bounds," IEEE Transactions on Information Theory, vol. 56, no. 10, pp. 5064-5082, Oct. 2010.

[14] H. L. Van Trees and K. L. Bell, Eds., Bayesian Bounds for Parameter Estimation and Nonlinear Filtering/Tracking. NewYork, NY, USA: Wiley/IEEE Press, Sep. 2007.

[15] H. L. Van Trees, Detection, Estimation and Modulation theory: Optimum Array Processing. New-York, NY, USA: John Wiley \& Sons, Mar. 2002, vol. 4.

[16] Y. Rockah and P. Schultheiss, "Array shape calibration using sources in unknown locations-part I: Far-field sources," IEEE Transactions on Acoustics, Speech, and Signal Processing, vol. 35, no. 3, pp. 286-299, Mar. 1987.
[17] H. Messer, "The hybrid Cramér-Rao lower bound - from practice to theory," in Proc. of IEEE Workshop on Sensor Array and Multi-channel Processing (SAM), Waltham, MA, USA, Jul. 2006, pp. 304-307.

[18] I. Reuven and H. Messer, "A Barankin-type lower bound on the estimation error of a hybrid parameter vector," IEEE Transactions on Information Theory, vol. 43, no. 3, pp. 1084-1093, May 1997.

[19] Y. Noam and H. Messer, "Notes on the tightness of the hybrid Cramér-Rao lower bound," IEEE Transactions on Signal Processing, vol. 57, no. 6, pp. 2074-2084, 2009.

[20] S. Bay, B. Geller, A. Renaux, J.-P. Barbot, and J.-M. Brossier, "On the hybrid Cramér-Rao bound and its application to dynamical phase estimation," IEEE Signal Processing Letters, vol. 15, pp. 453-456, 2008.

[21] K. Todros and J. Tabrikian, "Hybrid lower bound via compression of the sampled CLR function," in Proc. of IEEE Workshop on Statistical Signal Processing (SSP), Cardiff, Wales, UK, Aug. 2009, pp. 602-605.

[22] J. Tabrikian and J. L. Krolik, "Barankin bounds for source localization in an uncertain ocean environment," IEEE Transactions on Signal Processing, vol. 47, no. 11, pp. 2917-2927, Nov. 1999.

[23] S. Buzzi, M. Lops, and S. Sardellitti, "Further result on Cramér-Rao bounds for parameter estimation in long-code DS/CDMA systems," IEEE Transactions on Signal Processing, vol. 53, no. 3, pp. 1216-1221, Mar. 2005.

[24] P. Tichavský and K. Wong, "Quasi-fluid-mecanics based quasiBayesian Cramér-Rao bounds for towed-array direction finding," IEEE Transactions on Signal Processing, vol. 52, no. 1, pp. 36-47, Jan. 2007.

[25] A. Renaux, P. Forster, P. Larzabal, C. D. Richmond, and A. Nehorai, "A fresh look at the Bayesian bounds of the WeissWeinstein family," IEEE Transactions on Signal Processing, vol. 56, no. 11, pp. 5334-5352, Nov. 2008.

[26] E. L. Lehmann and G. Casella, Theory of Point Estimation, 2nd ed., ser. Springer Texts in Statistics. New-York, NY, USA: Springer, Sep. 2003.

[27] C. Ren, J. Galy, E. Chaumette, P. Larzabal, and A. Renaux, "A new class of hybrid lower bounds," in preparation for IEEE Transactions on Signal Processing. 